\title{
Protective effect of casticin in myocardial ischemia/ reperfusion injury in rats via attenuation of oxidative stress and inflammation
}

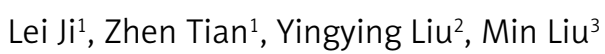

\author{
${ }^{1}$ Department of Cardiology, China-Japan Union Hospital of Jilin University, Changchun, \\ China \\ 2Department of Nephrology, China-Japan Union Hospital of Jilin University, Changchun, \\ China \\ ${ }^{3}$ Department of Geriatric Medicine, China-Japan Union Hospital of Jilin University, \\ Changchun, China
}

Submitted: 6 July 2020

Accepted: 17 September 2020

Arch Med Sci

DOI: https://doi.org/10.5114/aoms/127588

Copyright $\odot 2021$ Termedia \& Banach

\begin{abstract}
Introduction: Myocardial ischemic/reperfusion injury is the main prognostic factor after myocardial infarction. However, by the time reperfusion is achieved, the cardiac tissues have already undergone necrosis and initiated inflammation and oxidative stress. Therefore, the present study aimed to evaluate the beneficial effect of casticin (CST) on myocardial ischemia/ reperfusion $(\mathrm{MI} / \mathrm{R})$ injury and to explore its mechanism of action.

Material and methods: MI/R injury was induced using 30-min left anterior descending coronary artery (LAD) occlusion followed by $4 \mathrm{~h}$ reperfusion. CST was administered to rats before reperfusion. The outcome of CST was determined according to various indices of oxidative stress, inflammation, apoptotic genes and nuclear factor $\kappa$-light-chain-enhancer of activated $\mathrm{B}$ cells (NF-KB).

Results: The results suggested that CST causes significant improvement in left ventricular systolic pressure (LVSP) and derivative of pressure over time of maximal rate of rise of (usually) left ventricular pressure $\left( \pm d p / d t_{\max }\right)$ with reduction of left ventricular end-diastolic pressure (LVEDP). It also reduces increased ST segment together with restoration of the changes in RR interval and QRS complex. The histopathological analysis of cardiac tissue further corroborated the effect of CST. It also causes improvement of the antioxidant system by reducing malondialdehyde (MDA) level with increase in superoxide dismutase (SOD) and glutathione peroxidase (GPx). The levels of cytokines (TNF- $\alpha$, IL-1 $\beta$ and IL- 6 ) were also found to be reduced upon CST treatment. The expression of NF- $\kappa B$, inducible nitric oxide synthase (iNOS) and $B C L 2$-associated $X$ protein ( $\mathrm{Bax}$ ) was found to be reduced in the CST treated group together with an increase in $\mathrm{Bcl}-2$.

Conclusions: Collectively, the results of the study demonstrated that casticin protects rats from MI/R damage possibly via attenuation of oxidative stress, the inflammatory response and apoptosis. It was also shown to inhibit NF-KB and iNOS in western blot analysis.
\end{abstract}

Key words: myocardial ischemia, oxidative stress, inflammation, NF-kB, iNOS.

\section{Introduction}

Myocardial infarction (MI) is a devastating condition in which the heart is deprived of blood supply, which results in necrosis of cardio-

\author{
Corresponding author: \\ Min Liu PhD \\ Department of Geriatric \\ Medicine \\ China-Japan Union Hospital \\ of Jilin University \\ 130033, Changchun, China \\ e-mail: liumin01@jlu.edu.cn
}


myocytes [1]. The burden of MI can be easily understood by the fact that approximately 290 million patients with cardiovascular disease were reported in China, among whom 2.5 million were myocardial infarction patients in 2014 [2]. However, the necrosis of myocardial tissues could be prevented by restoring the lost blood supply, but reperfusion of the tissues further worsens the situation. This leads to another phenomenon known as myocardial ischemic/reperfusion (MI/R) injury where tissues and organs deteriorate before re-establishment of the blood supply $[3,4]$. In ischemic conditions, the cardiomyocytes and vascular endothelial cells undergo a series of numerous pathological events, such as production of reactive oxygen species (ROS). The high production of ROS causes generation of oxidative stress due to imbalance between the oxidative defense mechanism and induces lipid peroxidation of cellular membranes and release of various pro-inflammatory cytokines $[4,5]$. The complex etiology of $\mathrm{MI} / \mathrm{R}$ renders any single agent ineffective; thus, it is worthwhile to discover new agents which can provide protection against MI/R via multiple mechanisms.

Casticin (CST) is a polymethylflavone derived mainly from Vitex species of the family Verbenaceae. It shows a wide array of biological activities such as immunomodulatory, anti-hyperprolactinemia, anti-tumor, neuroprotective, anti-inflammatory and analgesic [6-8]. It has been found that CST causes blockade of nuclear factor $\kappa$-lightchain-enhancer of activated B cell (NF- $\kappa$ B) for antiinflammatory action [9]. Nevertheless, no study has been performed to evaluate the effect of CST on MI/R injury. Therefore, in the present study we wish to assess the effect of CST in experimentally induced MI/R injury and to elucidate the possible mechanism underlying its effect.

\section{Material and methods}

\section{Animals}

Adult male Sprague-Dawley (SD) (240-270 g) rats were selected for the study and housed in strict hygienic conditions after receiving them from the institutional animal house. The experiment was duly approved by the animal ethical committee of the institute and performed according to the national guidelines of animal care and use of China.

\section{Initiation of experimental myocardial ischemia/reperfusion injury}

The MI/R injury in rats was induced through blocking of the left anterior descending coronary artery (LAD) for $30 \mathrm{~min}$ with subsequent reperfusion for 24 h. Briefly, after anesthesia, the rats un- derwent tracheal intubation, and the LAD was obstructed using silk ligature for a period of $30 \mathrm{~min}$. After this, the ligature was removed and reperfusion was achieved for the next $24 \mathrm{~h}$. The same surgical procedure was used in the control except blocking of LAD. After $24 \mathrm{~h}$ of reperfusion or sham operation, the rats were executed for further analysis.

\section{Experimental design}

Thirty SD rats were taken and randomly divided into five groups containing six animals in each group: Group 1 - normal control, Group 2 - ISO rats; Group 3 - CST (10 mg/kg) + ISO, Group 4 - CST $(15 \mathrm{mg} / \mathrm{kg})+$ ISO, Group 5 - CST $(20 \mathrm{mg} / \mathrm{kg})+$ ISO

Casticin in the dosage as indicated above after being dissolved in normal saline (also 1\% DMSO) was administered to rats 30 min before the induction of myocardial ischemia injury via intraperitoneal injection. At the end of the experiment, hemodynamic parameters were recorded as indicated below. Thereafter, the rats were killed under anesthetized conditions using urethane $(1 \mathrm{~g} / \mathrm{kg}$, intra-peritoneally injected) to isolate blood. The heart tissues were rinsed in ice-cold $0.9 \% \mathrm{NaCl}$ after harvesting.

\section{Hemodynamic measurements}

The hemodynamic parameters such as systolic, diastolic and mean arterial blood pressure (SAP, DAP and MAP, respectively) as well as HR were recorded using CARDIOSYSCO-101 (Experimentria, Hungary) with a pressure transducer connected to the right carotid artery.

\section{Analysis of electrocardiogram and cardiac functional parameters}

Electrocardiogram (ECG) recordings were obtained from the animals using a BL-420S biological function experimental system. For ECG recording, the standard limb lead II of the surface ECG was used for each rat by the PowerLab Data Acquisition System (AD Instruments, Australia) connected to a computer enabled with LabChart professional software version 8 (AD Instruments, Australia).

The ECG record exhibits the following parameters: heart rate in beats per minute, ST height, RR, and QRS complex.

\section{Determination of serum lactate dehydrogenase and creatine kinase levels}

The levels of serum lactate dehydrogenase $(\mathrm{LDH})$ and serum creatine kinase (CK) myocardial band were recorded using commercial assay kits obtained from Nanjing Jiancheng Bioengineering Institute, China as per the given instructions. 
Determination of tumor necrosis factor- $\alpha$, cardiac troponin T and interleukin-6 levels in cardiac tissues

The serum levels of tumor necrosis factor- $\alpha$ (TNF- $\alpha$ ) and interleukin (IL) 6 in were determined using commercial sandwich enzyme-linked immunosorbent assay (ELISA) kits obtained from Nanjing Jiancheng Bioengineering Institute, China. Moreover, a cardiac troponin T (Tn-T) ELISA kit (cat. No. ARB13662) was obtained from Rapidbio, West Hills, CA, USA [10-13].

\section{Evaluation of lipid peroxidation and antioxidant enzyme levels}

The supernatant of heart tissue homogenates was used to assay MPO (cat. no. A044), malondialdehyde (MDA) level (cat. no. A003-2), and superoxide dismutase (SOD) (cat. no. A001-3) and glutathione peroxidase (GPx) (cat. no. A005) activities as per the manufacturer's instructions (Nanjing Jiancheng Bioengineering Institute, China) on a microplate reader (560 and $532 \mathrm{~nm}$ ).

\section{Western blot analysis}

Using ice-cold RIPA lysis buffer, total protein was extracted. The dissolved proteins were then A

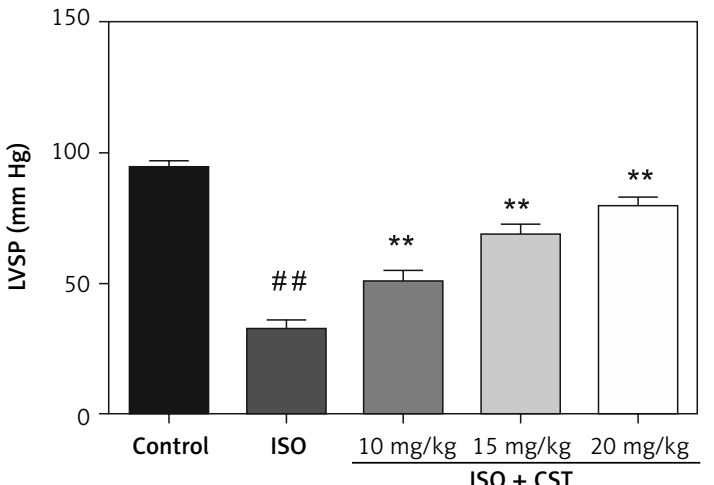

C

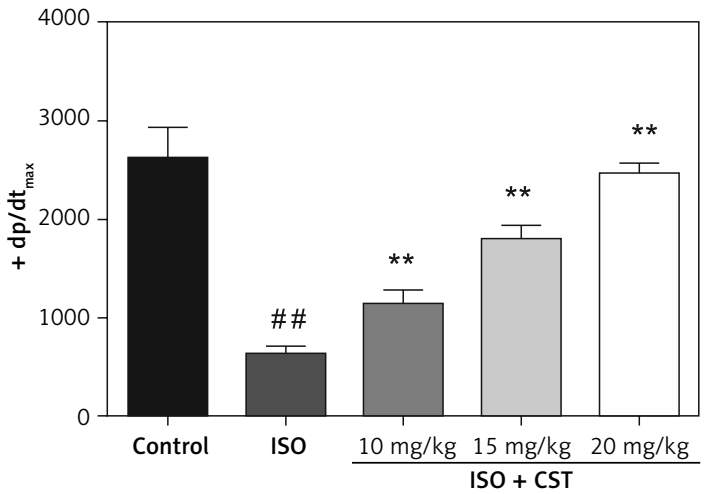

centrifuged at $12,000 \mathrm{rpm}$ for $5 \mathrm{~min}$ at $4^{\circ} \mathrm{C}$ and the bicinchoninic acid protein assay (BCA) was used to determine the total protein concentration. The isolated proteins were loaded on SDS-PAGE and transferred onto the PVDF membrane and further surveyed with primary antibody. The expression of proteins was detected using an enhanced chemiluminescence reagent after incubating for $1 \mathrm{~h}$ at room temperature with HRP-conjugated goat antirabbit IgG. Quantification of protein bands was performed using ImageJ v1.42q software (National Institutes of Health, Bethesda, MA, USA).

\section{Statistical analysis}

Data were analyzed by SPSS 17.0 software and expressed as the means \pm SEM. Differences were analyzed by one-way analysis of variance (ANOVA) followed by Dunnett's test for individual comparisons between group means. A $p$-value of 0.05 was considered statistically significant.

\section{Results}

Effect of CST on hemodynamic parameters in MRI rats

Initially, the effect of CST on left ventricular function in rats was determined. As shown in Figure 1,

\section{B}
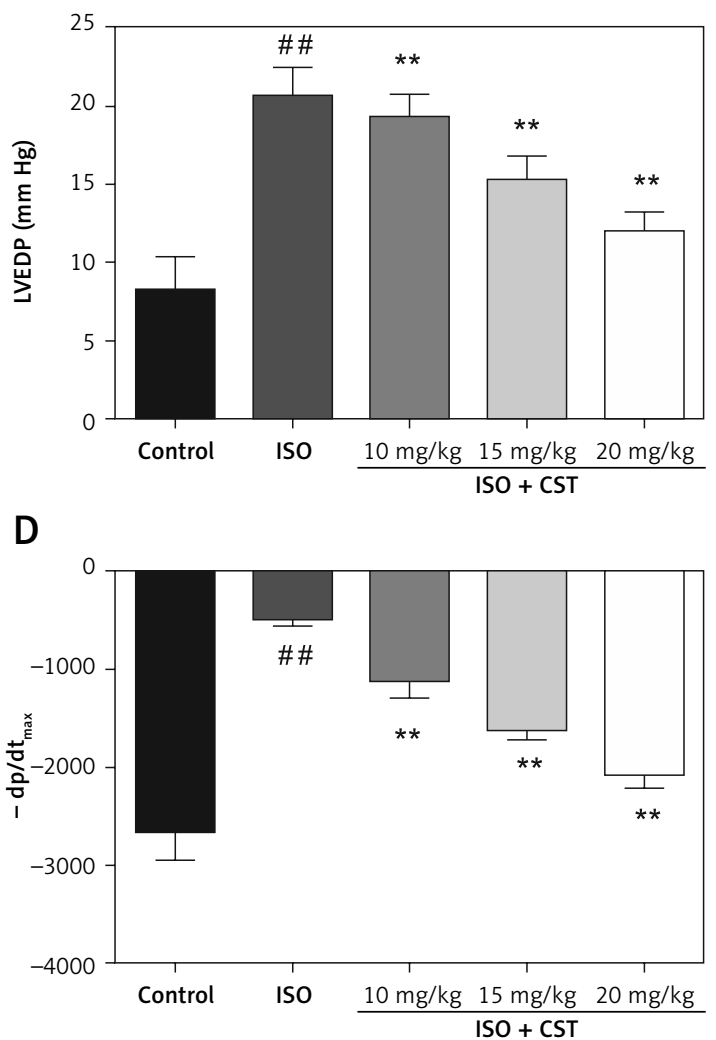

Figure 1. Effect of CST on hemodynamic parameters of MRI rats, (A) LVSP (B) LVEDP, (C and D) $\pm d p / d t_{\text {max }}$. Values represent the mean $\pm \mathrm{SD}$ and are representative of three independent experiments

${ }^{*} p<0.05$ vs control; ${ }^{*} p<0.05,{ }^{* *} p<0.01$ vs model. 
A

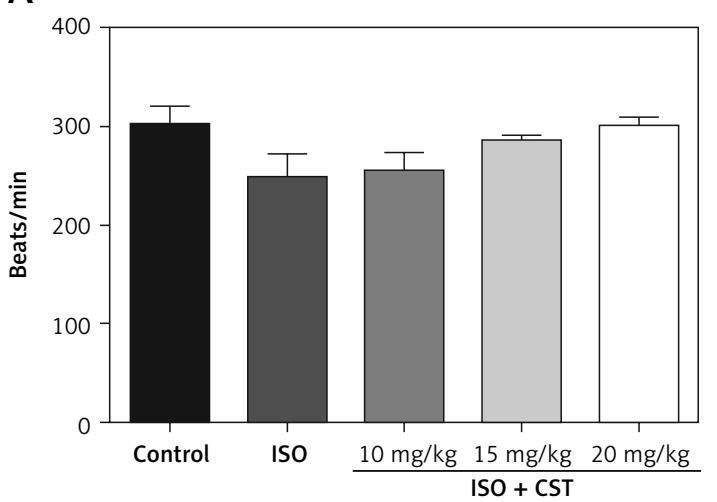

C

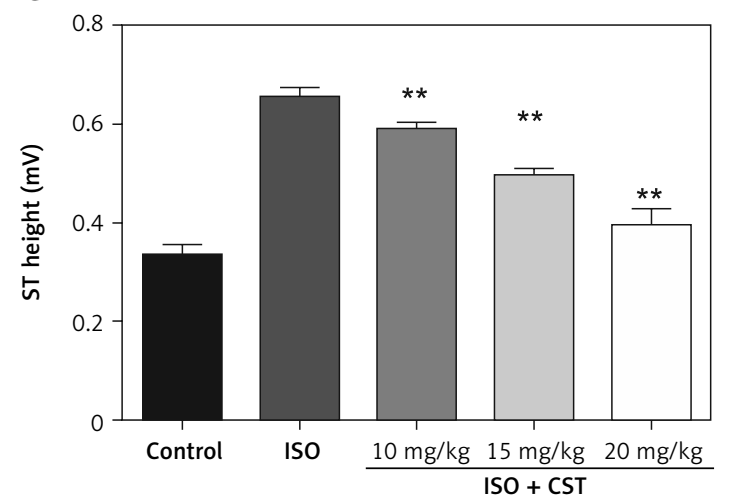

E

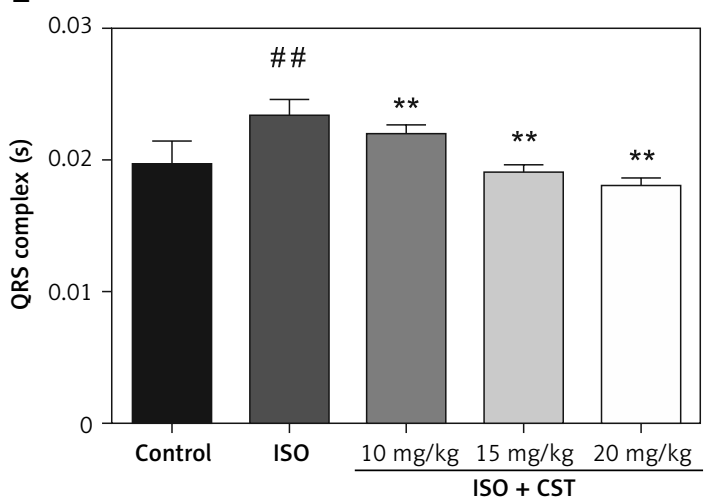

B

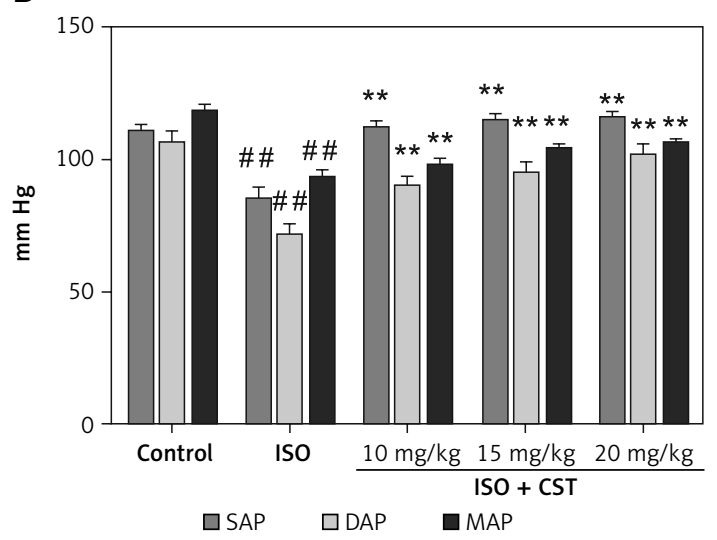

D

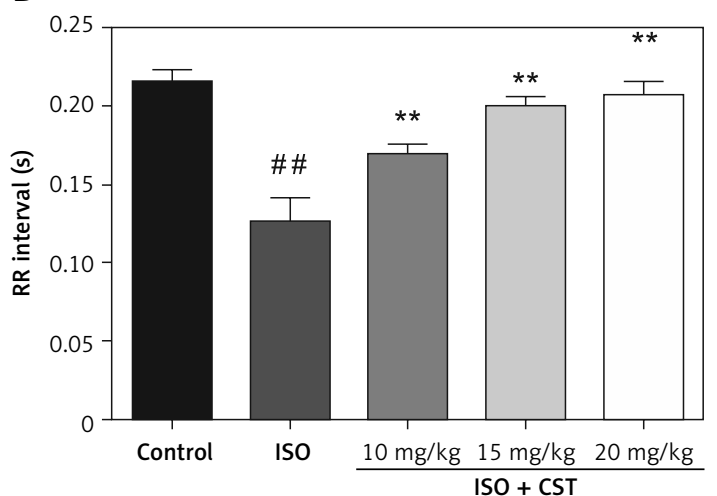

F

Control Model

ISO + CST $20 \mathrm{mg} / \mathrm{kg}$

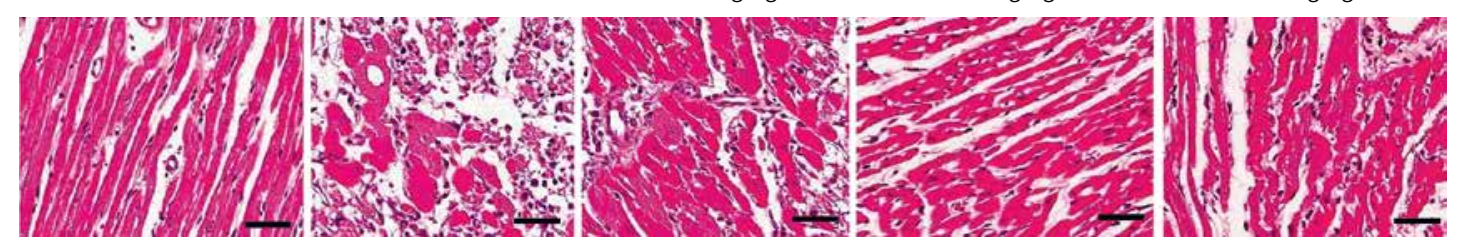

Figure 2. Effect of CST on (A) heart beat and (B) arterial pressure and ECG of rats (C) ST height, (D) RR interval, (E) ORS complex, and (F) cardiac histopathology. Values represent the mean \pm SD and are representative of three independent experiments

${ }^{\# \#} p<0.05$ vs control; ${ }^{*} p<0.05,{ }^{* *} p<0.01$ vs model. 
CST causes significant improvement in left ventricular systolic pressure (LVSP) and derivative of pressure over time of maximal rate of rise of (usually) left ventricular pressure $\left( \pm \mathrm{dp} / \mathrm{dt}_{\max }\right)$ with reduction of left ventricular end-diastolic pressure (LVEDP) in MRI rats.

Moreover, CST also causes improvement of heart beat and arterial pressure in a concentration-dependent manner; see Figures $2 \mathrm{~A}$ and $\mathrm{B}$, respectively. In ECG analysis as shown in Figures 2 C, D and $E$, the ISOtreated rats showed amplified heart rate, elevated ST segment, merged T and P waves, flutter waves, decreased RR interval, and widening of QRS complex. In contrast, in the CST treated group, ST segment elevation was reduced together with restoration of the changes in RR interval and QRS complex.

\section{Effect of CST on cardiac histopathology}

The effect of CST on the histopathology of cardiac tissue of MRI rats was also investigated to determine its effect at the micro-structural level. As can be seen in Figure $2 \mathrm{~F}$, the control group showed homogeneous and intact histo-architecture with no evidence of cell death or inflammation. However, the model group showed apoptosis of muscle fibers with edema. Necrosis was found to be reduced in a concentration-dependent man-

A

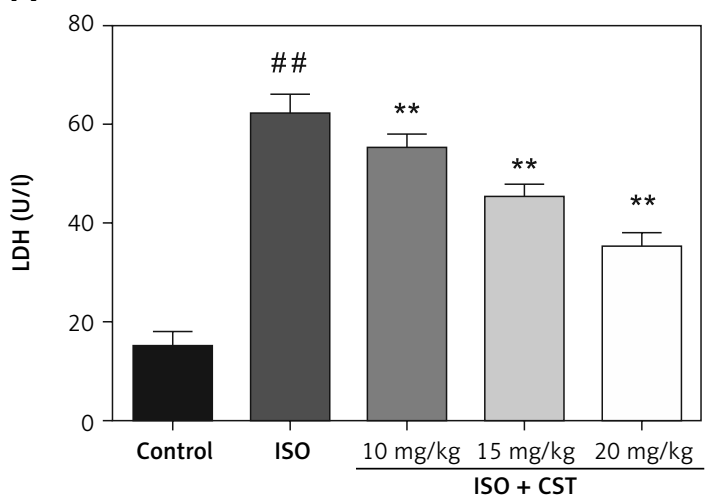

C

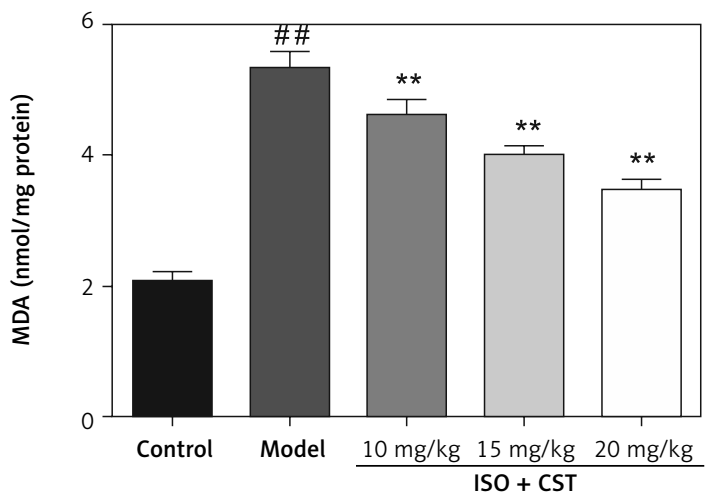

ner with reduction of edema and myocyte proliferation.

This observation confirmed that CST could mitigate the after-effects of MRI in rats.

\section{Effect of CST on myocardial injury biomarkers}

The effect of CST on the serum biomarkers linked with cardiac injury (LDH and CK) was investigated.

As can be seen in Figures 3 A and B, ISO-treated rats showed increased levels of $\mathrm{LDH}$ and $\mathrm{CK}$, which were significantly reduced upon administration of CST in CST treated groups.

\section{Effect of CST on oxidative stress biomarkers}

The effect of CST on various biomarkers of oxidative stress was quantified, as presented in Figures 3 C, D and E. The disease model group showed an increased level of MDA with a reduced level of SOD and GPX as compared with the control group.

Moreover, in the CST treated group, the level of these biomarkers of oxidative stress was restored approximately to normal in a concentration dependent manner.

\section{B}

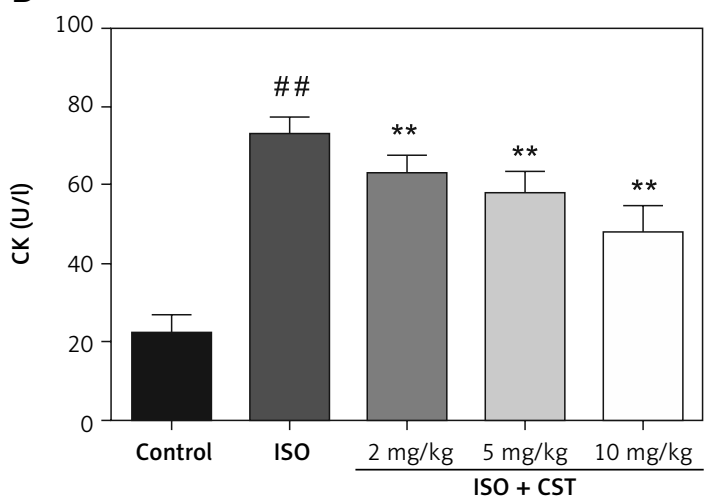

D

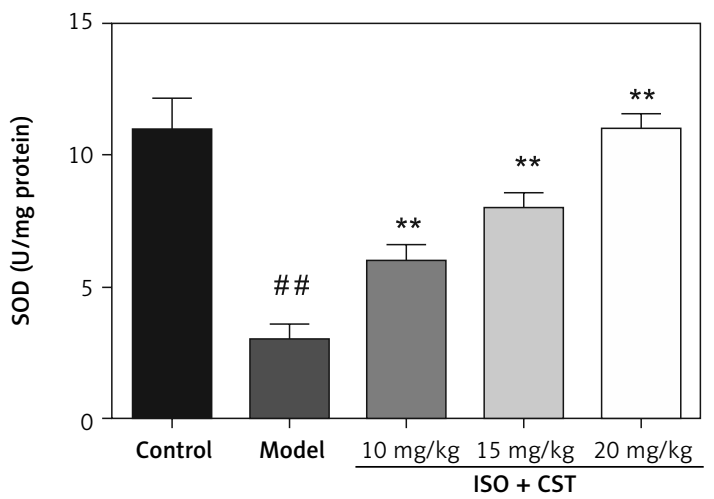

Figure 3. Effect of CST on cardiac injury biomarkers, (A) LDH (B) CK; indices of oxidative stress (C) MDA, (D) SOD ${ }^{\# *} p<0.05$ vs control; ${ }^{*} p<0.05,{ }^{* *} p<0.01$ vs model 

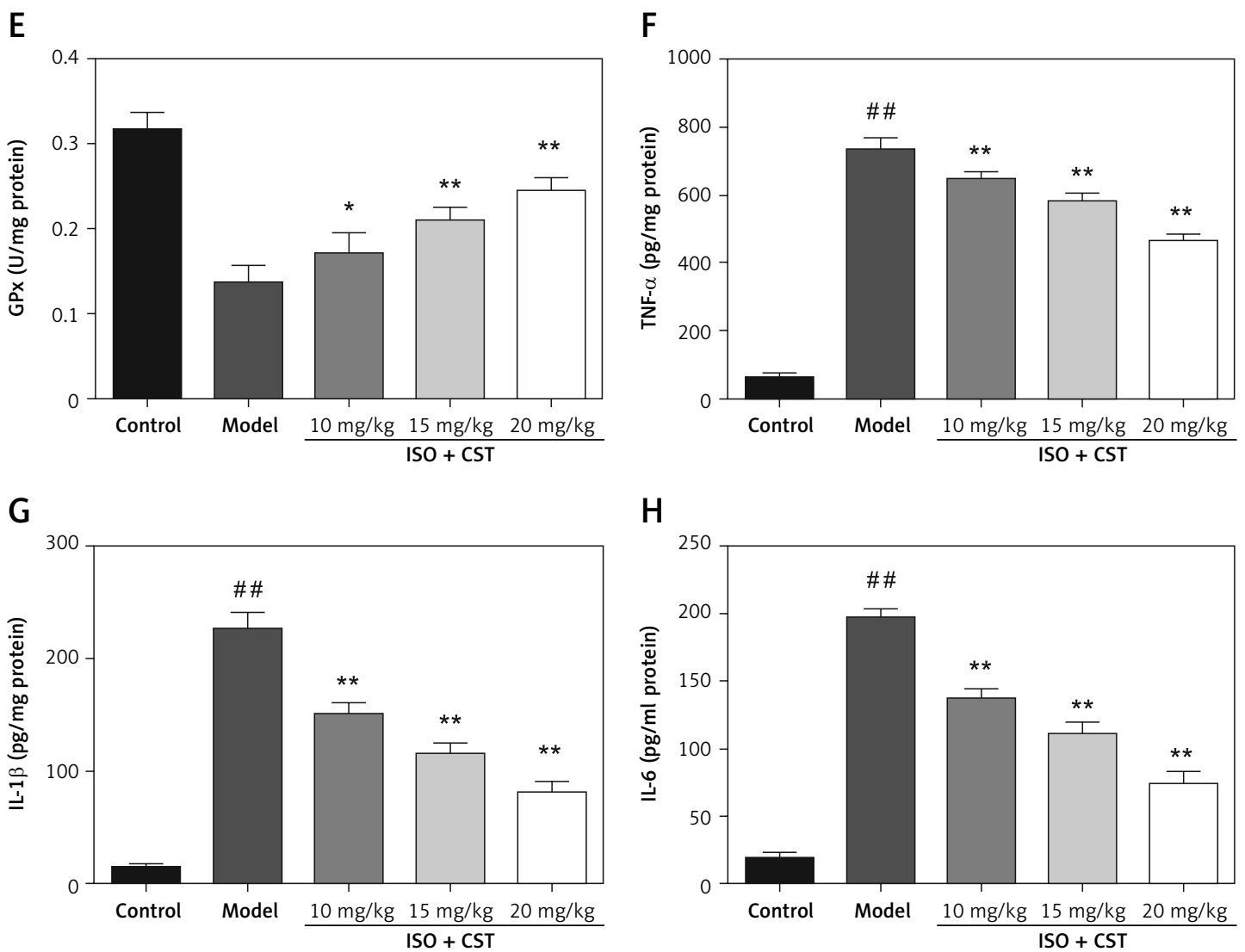

Figure 3. Cont. (E) GPx; and inflammatory cytokines (F) TNF- $\alpha$, (G) IL-1 $1 \beta$ and (H) IL-6. Values represent the mean $\pm \mathrm{SD}$ and are representative of three independent experiments

${ }^{\# \#} p<0.05$ vs control; ${ }^{*} p<0.05,{ }^{* *} p<0.01$ vs model.

A

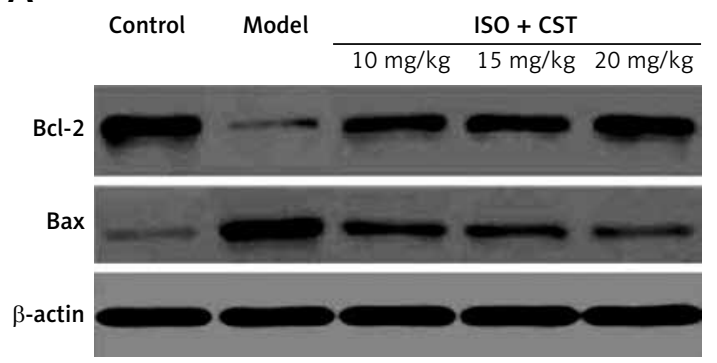

C

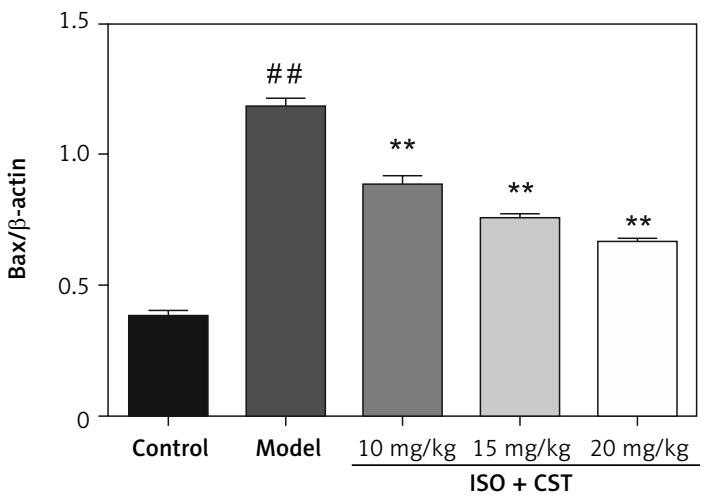

B

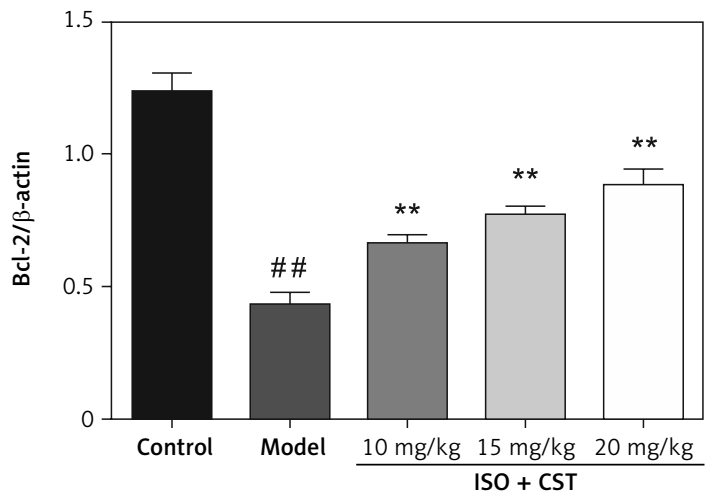

Figure 4. Effect of CST on (A) BCl-2 and Bax using western blot, densitometry data of (B) Bcl-2 and (C) Bax. Values represent the mean \pm SD and are representative of three independent experiments ${ }^{\# *} p<0.05$ vs control; ${ }^{*} p<0.05,{ }^{* *} p<0.01$ vs model. 


\section{Effect of CST on pro-inflammatory cytokines}

As shown in Figures $3 \mathrm{~F}, \mathrm{G}$ and $\mathrm{H}$, the cytokines (TNF- $\alpha$, IL-1 $\beta$ and IL-6) were increased in the model group as compared to the control. However, the level of these cytokines was significantly reduced in the CST treated group in a dose-dependent manner. It could suggest that CST had an inhibitory effect on the increased level pro-inflammatory cytokines.

\section{Effect of CST on apoptotic genes}

The effect of CST on genes which are known to induce pro-apoptotic and apoptotic action was investigated. As presented in Figure 4, the level of $\mathrm{Bcl}-2$ was found to be decreased with an increase in BCL2-associated $X$ protein (Bax) in the model group as compared to the control. Moreover, the levels of these genes were found to be restored to near normal as compared to the model group. This suggests that CST can inhibit apoptosis of myocardial tissues under MI/R injury.

\section{Effect of CST on NF- $\kappa B$ and iNOS}

In order to understand the mechanism of action, the effect of CST on NF- $\kappa B$ and inducible

A

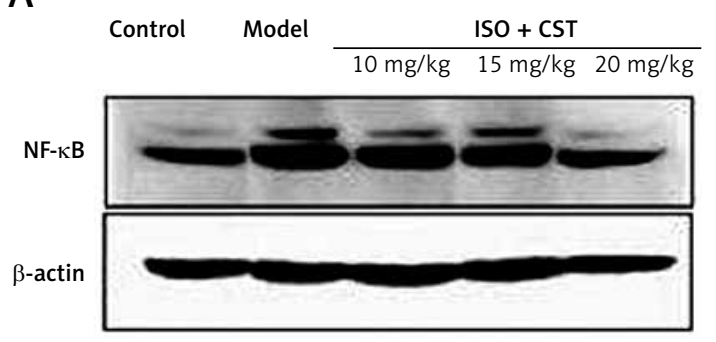

C

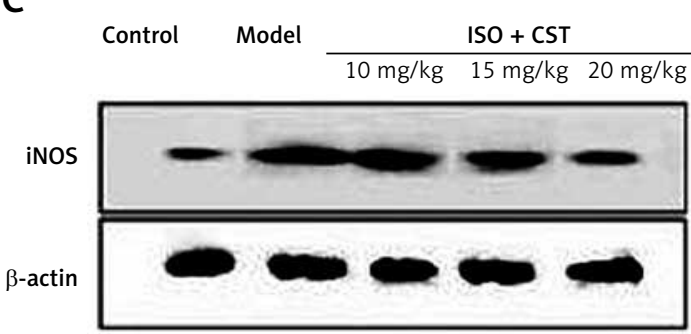

nitric oxide synthase (iNOS) was investigated by western blot analysis. As shown in Fig. 5, the expression of both NF- $\mathrm{KB}$ and iNOS was found to be increased in the model group in comparison to the control. Moreover, the levels of these biomarkers were found to be reduced significantly in the CST treated group in a concentration-dependent manner.

\section{Discussion}

Sustained myocardial ischemia-reperfusion induces injury to cardiomyocytes and initiates various forms of cell death that contribute to myocardial infarction. Myocardial ischemia-reperfusion also induces injury to the coronary microcirculation, including capillary rupture and hemorrhage [14]. The present study showed that casticin could effectively mitigate the biochemical alteration in MRI rats. The results suggested that casticin improved the blood supply together with reduction of oxidative stress, inflammation and apoptosis via inhibition of NF- $\kappa$ B and iNOS.

After MI, the key aim of the therapeutics is to restore the blood supply by improving the blood flow [15]. In the present study, it was found that casticin caused improvement in LVSP and $\pm \mathrm{dp} / \mathrm{dt}_{\max }$ with reduction of LVEDP in MRI rats. It also im-

B

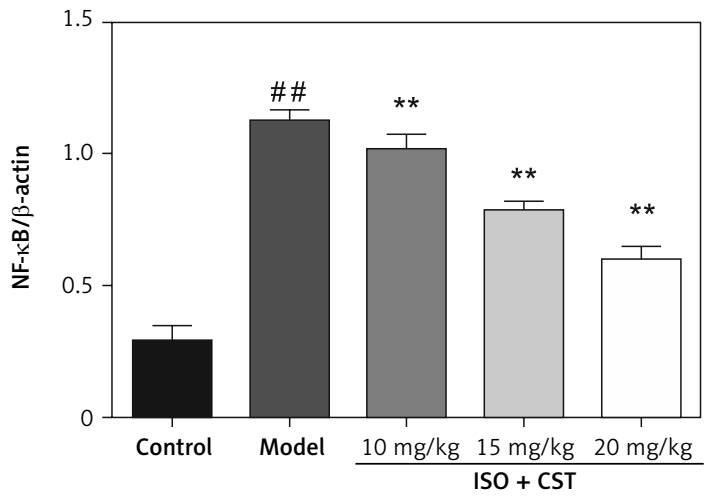

D

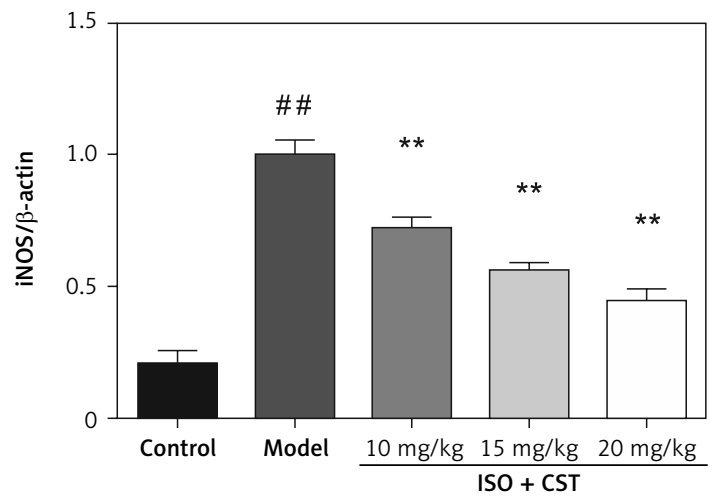

Figure 5. Effect of CST on expression of ( $\mathbf{A}$ and $\mathbf{B}$ ) NF-KB and (C and $\mathbf{D}$ ) iNOS by western blot analysis. Values represent the mean $\pm \mathrm{SD}$ and are representative of three independent experiments

${ }^{\# *} p<0.05$ vs control; ${ }^{*} p<0.05,{ }^{* *} p<0.01$ vs model. 
proved the RR interval and QRS complex with reduction of ST segment elevation. Studies have shown the clinical significance of LDH and CK as vital biomarkers to predict cardiac injury. The results suggested that casticin causes significant reduction of both LDH and CK levels in cardiac tissue of rats. The histopathology of rats was also found to be in agreement with the above observation, where casticin causes reduction of the necrosis of myocardial tissue with reduction of infiltration. These results indicated that casticin may have a cardioprotective effect against MI/R injury.

Prompted by the above results, in the next part, we aimed to investigate the probable mechanism underlying the cardioprotective effect of casticin. Studies have shown that oxidative stress has a very deleterious effect on the recovery from MI/R injury [16-20]. It induces production of an excessive amount of reactive oxygen species in the impaired anti-oxidant defense system [21-23]. It has been suggested that these free radicals cause degradation of DNA, lipids, and membranes, which promotes tissue necrosis [21, $24,25]$. Thus agents improving the antioxidant system showed benefit against myocardial ischemia/reperfusion. In the present study, we observed that casticin induced significant reduction of MDA production and restored the level of SOD and GPx in cardiac tissue. Thus, it could be suggested that casticin protects against myocardial damage possibly by restraining the production of free radical via improving the anti-oxidant mechanism.

Studies have shown that oxidative stress promotes inflammation in myocardial tissues [26-30]. It promotes the generation of various pro-inflammatory cytokines regulated via acti-

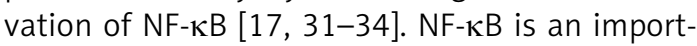
ant inflammatory mediator which promotes the transcription of various genes after activation by numerous stimuli responsible for cellular viability, proliferation, apoptosis and the inflammatory response. The activation of NF- $\kappa B$ is generally mediated via ligands of various cytokine receptors, pattern-recognition receptors (PRRs), TNF receptor (TNFR) superfamily members, as well as T-cell receptor (TCR) and B-cell receptor [35-38]. Upon activation NF- $\kappa B$ dissociates from its inhibitory regulator $1 \kappa B-\alpha$ and translocates in free form into the nucleus from the cytoplasm $[39,40]$. In the present study, we found that casticin causes reduction of NF- $\kappa B$ in myocardial tissues together with iNOS. iNOS is another important mediator found overexpressed in myocardial tissue. A high level of iNOS promotes excessive production of nitric oxide for a prolonged duration. It also induces oxyradical-mediated myocardial damage or acts as a negative inotrope due to myocardi- al cGMP production [41-44]. Numerous studies have shown the involvement of apoptosis or planned cell death of cardiomyocytes in MI/R in jury [45-49]. The $\mathrm{Bcl}-2$ family genes are notably acknowledged as the main regulators of apoptosis. $\mathrm{Bcl}-2$ was shown to inhibit the process of apoptosis, whereas Bax acts as a pro-apoptotic gene; thus the intricate balance between these two genes regulates apoptosis [50]. In the present study, casticin caused reduction of Bax, and increased $\mathrm{Bcl}-2$ in a dose-dependent manner. Thus, the above observations suggest that casticin exerts a cardioprotective effect via inhibition of inflammation and apoptosis.

In conclusion, it could be suggested that casticin protects the heart after MI/R injury in rats via multiple mechanisms, such as reduction of oxidative stress, inflammation, and apoptosis and improvement of hemodynamic status. The present study may serve as a template for further studies on casticin application against MI/R injury.

\section{Conflict of interest}

The authors declare no conflict of interest.

\section{References}

1. Reed GW, Rossi JE, Cannon CP. Acute myocardial infarction. Lancet 2017; 389: 197-210.

2. Chen WW, Gao RL, Liu LS, et al. China cardiovascular diseases report 2015: a summary. I Geriatr Cardiol 2017; 14: 1-10.

3. Hausenloy DJ, Yellon DM. Myocardial ischemia-reperfusion injury: A neglected therapeutic target. J Clin Invest 2013; 123: 92-100.

4. Buja LM. Myocardial ischemia and reperfusion injury. Cardiovasc Pathol 2005; 14: 170-5.

5. Jennings RB. Historical perspective on the pathology of myocardial ischemia/reperfusion injury. Circ Res 2013; 113: 428-38.

6. Zahid H, Rizwani GH, Ishaqe S. Phytopharmacological review on vitex agnus-castus: a potential medicinal plant. Chinese Herb Med 2016; 8: 24-9.

7. Chan EWC, Wong SK, Chan HT. Casticin from Vitex species: a short review on its anticancer and anti-inflammatory properties. J Integr Med 2018; 16: 147-52.

8. Chen SN, Friesen JB, Webster D, et al. Phytoconstituents from Vitex agnus-castus fruits. Fitoterapia 2011; 82: 528-33.

9. Liou CJ, Bin Len W, Wu SJ, Lin CF, Wu XL, Huang WC. Casticin inhibits COX-2 and iNOS expression via suppression of NF-KB and MAPK signaling in lipopolysaccharide-stimulated mouse macrophages. J Ethnopharmacol 2014; 158 Pt A: 310-6.

10. Sampath PD, Vijayaraghavan K. Cardioprotective effect of $\alpha$-mangostin, a xanthone derivative from Mangosteen on tissue defense system against isoproterenol-induced myocardial infarction in rats. J Biochem $\mathrm{Mol}$ Toxicol 2007; 21: 336-9.

11. Yousefi K, Fathiazad F, Soraya H, Rameshrad M, MalekiDizaji N, Garjani A. Marrubium vulgare L. methanolic extract inhibits inflammatory response and prevents cardiomyocyte fibrosis in isoproterenol-induced 
acute myocardial infarction in rats. Bioimpacts 2014; 4: 21-7.

12. Nagoor Meeran MF, Jagadeesh GS, Selvaraj P. Thymo attenuates inflammation in isoproterenol induced myocardial infarcted rats by inhibiting the release of lysosomal enzymes and downregulating the expressions of proinflammatory cytokines. Eur J Pharmacol 2015; 754: 153-61.

13. Yang J, Wang HX, Zhang YJ, et al. Astragaloside IV attenuates inflammatory cytokines by inhibiting TLR4/NF-kB signaling pathway in isoproterenol-induced myocardial hypertrophy. J Ethnopharmacol 2013; 150: 1062-70.

14. Heusch G. Myocardial ischaemia-reperfusion injury and cardioprotection in perspective. Nat Rev Cardiol 2020; 17: 773-89.

15. Frank A, Bonney M, Bonney S, Weitzel L, Koeppen M, Eckle T. Myocardial ischemia reperfusion injury: from basic science to clinical bedside. Semin Cardiothorac Vasc Anesth 2012; 16: 123-32.

16. Misra MK, Sarwat M, Bhakuni P, Tuteja R, Tuteja N. Oxidative stress and ischemic myocardial syndromes. Med Sci Monit 2009; 15: RA209-19.

17. Gill R, Tsung A, Billiar T. Linking oxidative stress to inflammation: Toll-like receptors. Free Radic Biol Med 2010; 48: 1121-32.

18. Kurian GA, Rajagopal R, Vedantham S, Rajesh M. The role of oxidative stress in myocardial ischemia and reperfusion injury and remodeling: revisited. Oxid Med Cell Longev 2016; 2016: 1656450.

19. Hashmi S, Al-Salam S. Acute myocardial infarction and myocardial ischemia-reperfusion injury: a comparison. Int J Clin Exp Pathol 2015; 8: 8786-96.

20. Gottlieb RA. Cell death pathways in acute ischemia/ reperfusion injury. J Cardiovasc Pharmacol Ther 2011; 16: 233-8.

21. Giordano FJ. Oxygen, oxidative stress, hypoxia, and heart failure. J Clin Invest 2005; 115: 500-8.

22. Tsutsui $H$, Kinugawa S, Matsushima S. Oxidative stress and heart failure. Am J Physiol Hear Circ Physiol 2011; 301: 2181-90

23. Dhalla NS, Temsah RM, Netticadan T. Role of oxidative stress in cardiovascular diseases. J Hypertens 2000; 18: 655-73.

24. Mei Y, Thompson MD, Cohen RA, Tong XY. Autophagy and oxidative stress in cardiovascular diseases. Biochim Biophys Acta 2015; 1852: 243-51.

25. Briones AM, Touyz RM. Oxidative stress and hypertension: current concepts. Curr Hypertens Rep 2010; 12: 135-42.

26. Wang TY, AlJaroudi WA, Newby LK. Markers of cardiac ischemia and inflammation. Cardiol Clin 2005; 23 : 491-501.

27. Burke AP, Virmani R. Pathophysiology of acute myocar dial infarction. Med Clin North Am 2007; 91: 553-72.

28. Entman ML, Michael L, Rossen RD, et al. Inflammation in the course of early myocardial ischemia. FASEB J 1991, 5: 2529-37.

29. Rohrbach S, Troidl C, Hamm C, Schulz R. Ischemia and reperfusion related myocardial inflammation: A network of cells and mediators targeting the cardiomyocyte. IUBMB Life 2015; 67: 110-9.

30. Bartekova M, Radosinska J, Jelemensky M, Dhalla NS. Role of cytokines and inflammation in heart function during health and disease. Heart Fail Rev 2018; 23: 733-58.

31. Turer AT, Hill JA. Pathogenesis of myocardial ischemia-reperfusion injury and rationale for therapy. Am J Cardiol 2010; 106: 360-8.
32. Paoletti R, Gotto AM, Hajjar DP. Inflammation in atherosclerosis and implications for therapy. Circulation 2004; 109 (23 Suppl 1): III20-6.

33. Yoo SY, Kwon SM. Angiogenesis and its therapeutic opportunities. Mediators Inflamm 2013; 2013: 127170

34. Arumugam TV, Okun E, Tang SC, Thundyil J, Taylor SM, Woodruff TM. Toll-like receptors in ischemia-reperfusion injury. Shock 2009; 32: 4-16.

35. Liu T, Zhang L, Joo D, Sun SC. NF-KB signaling in inflammation. Signal Transduct Target Ther 2017; 2: 17023.

36. Newton K, Dixit VM. Signaling in innate immunity and inflammation. Cold Spring Harb Perspect Biol 2012; 4: a006049.

37. Lawrence T. The nuclear factor NF-kappa B pathway in inflammation. Cold Spring Harb Perspect Biol 2009; 6: a001651.

38. Ghosh S, Hayden MS. New regulators of NF-KB in inflammation. Nat Rev Immunol 2008; 8: 837-48.

39. Sun SC. The non-canonical NF- $\mathrm{KB}$ pathway in immunity and inflammation. Nat Rev Immunol 2017; 17: 545-58.

40. Rahman A, Fazal F. Blocking NF-kB: an inflammatory issue. Proc Am Thorac Soc 2011; 8: 497-503.

41. Korhonen R, Lahti A, Kankaanranta H, Moilanen E. Nitric oxide production and signaling in inflammation. Curr Drug Targets Inflamm Allergy 2005; 4: 471-9.

42. Lechner M, Lirk P, Rieder J. Inducible nitric oxide synthase (iNOS) in tumor biology: the two sides of the same coin. Semin Cancer Biol 2005; 15: 277-89.

43. Jaiswal M, LaRusso NF, Burgart LJ, Gores GJ. Inflammatory cytokines induce DNA damage and inhibit DNA repair in cholangiocarcinoma cells by a nitric oxide-dependent mechanism. Cancer Res 2000; 60: 184-90.

44. Lirk P, Hoffmann G, Rieder J. Inducible nitric oxide synthase - time for reappraisal. Curr Drug Targets Inflamm Allergy 2002; 1: 89-108.

45. Zhao ZQ Vinten-Johansen J. Myocardial apoptosis and ischemic preconditioning. Cardiovasc Res 2002; 55: 438-55.

46. Freude B, Masters TN, Robicsek F, et al. Apoptosis is initiated by myocardial ischemia and executed during reperfusion. J Mol Cell Cardiol 2000; 32: 197-208.

47. Kubasiak LA, Hernandez OM, Bishopric NH, Webster KA. Hypoxia and acidosis activate cardiac myocyte death through the Bcl-2 family protein BNIP3. Proc Natl Acad Sci USA 2002; 99: 12825-30.

48. Eken MK, Ersoy GS, Kaygusuz El, et al. Etanercept protects ovarian reserve against ischemia/reperfusion injury in a rat model. Arch Med Sci 2019; 15: 1104-12.

49. Sedaghat Z, Kadkhodaee M, Seifi B, Salehi E. Inducible and endothelial nitric oxide synthase distribution and expression with hind limb per-conditioning of the rat kidney. Arch Med Sci 2019; 15: 1081-91.

50. Anselmi A, Abbate A, Girola F, et al. Myocardial ischemia, stunning, inflammation, and apoptosis during cardiac surgery: a review of evidence. Eur J Cardiothorac Surg 2004; 25: 304-11. 